\title{
Error Bound for Conic Inequality in Hilbert Spaces
}

\author{
Jiangxing Zhu, Qinghai He, and Jinchuan Lin \\ Department of Mathematics, Yunnan University, Kunming 650091, China \\ Correspondence should be addressed to Qinghai He; heqh@ynu.edu.cn \\ Received 15 February 2014; Accepted 23 March 2014; Published 15 April 2014 \\ Academic Editor: Jen-Chih Yao
}

Copyright (C) 2014 Jiangxing Zhu et al. This is an open access article distributed under the Creative Commons Attribution License, which permits unrestricted use, distribution, and reproduction in any medium, provided the original work is properly cited.

We consider error bound issue for conic inequalities in Hilbert spaces. In terms of proximal subdifferentials of vector-valued functions, we provide sufficient conditions for the existence of a local error bound for a conic inequality. In the Hilbert space case, our result improves and extends some existing results on local error bounds.

\section{Introduction}

Let $X$ be a Banach space and let $f: X \rightarrow \overline{\mathbb{R}}:=\mathbb{R} \cup\{+\infty\}$ be a proper lower semicontinuous function. Consider the following inequality:

$$
f(x) \leq 0
$$

Let $S_{f}:=\{x \in X: f(x) \leq 0\}$ and $a \in S_{f}$. Recall that inequality (IE) has a local error bound at $a$ if there exist $\tau, \delta \in(0,+\infty)$ such that

$$
d\left(x, S_{f}\right) \leq \tau[f(x)]_{+} \quad \forall x \in B(a, \delta),
$$

where $d\left(x, S_{f}\right):=\inf \left\{\|x-u\|: u \in S_{f}\right\},[f(x)]_{+}:=$ $\max \{f(x), 0\}=d\left(0, f(x)+\mathbb{R}_{+}\right)$and $B(a, \delta)$ denotes the open ball centered at $a$ of radius $\delta$. In the variational analysis literature, sensitivity analysis of mathematical programming and convergence analysis of some algorithms of optimization problems are deeply tied to the notion of error bound. Since Hoffman's pioneering work [1], the study of error bounds has received extensive attention in the mathematical programming literature (for details, see [2-9] and references therein). For us, the work of Ioffe in this area will be particularly important. The author in his seminal work [10] first characterized error bound (under a different name) in terms of the subdifferentials and gave the following interesting result: if $f$ is locally Lipschitz at $a \in S_{f}$ and there exist $\eta, \delta \in(0,+\infty)$ such that

$$
\eta \leq d(0, \partial f(x)) \quad \forall x \in B(a, \delta) \backslash S_{f},
$$

then (IE) has a local error bound at $a$, where $\partial f(x)$ denotes the Clarke-Rockafellar subdifferential of $f$ at $x$ (for its definition, see Section 2). Results of the same flavour have sprung up. In 2010, in considering a general closed multifunction $F$ in place of $f$ and with the subdifferential $\partial f(x)$ replaced by the coderivative of $F$, Zheng and $\mathrm{Ng}$ [11] extended the above-mentioned result to a generalized equation defined by a closed multifunction. Recently, Zheng and $\mathrm{Ng}$ [12] once again extended Ioffe's classic result to the conic inequality case in Asplund spaces in terms of the conic subdifferential $\widehat{\partial}_{K} \Phi$ defined by Fréchet normal cone. In this paper, we will extend Ioffe's result to the conic inequality case in the Hilbert space setting.

Let $X, Y$ be Banach spaces with $Y$ ordered by a closed convex cone $K \subset Y$. Let $b \in Y$ and $\Phi: X \rightarrow Y^{\bullet}$ be a function, where $Y^{\bullet}$ is the union of $Y$ with an abstract infinity $\infty_{Y}$. In this paper, we consider the following conic inequality:

$$
\Phi(x) \leq_{K} b .
$$

Indeed, many constraint systems in optimization can be viewed as special cases of (CIE). For example, when $Y=$ $\mathbb{R}^{n+m}, \mathrm{~K}=\mathbb{R}_{+}^{n} \times\{0\}$, and $\Phi(x)=\left(\phi_{1}(x), \ldots, \phi_{n+m}(x)\right),(\mathrm{CIE})$ reduces to

$$
\begin{gathered}
\phi_{i}(x) \leq 0 \text { for } i=1,2, \ldots, n, \\
\phi_{i}(x)=0 \text { for } i=n+1, \ldots, n+m,
\end{gathered}
$$

where each $\phi_{i}$ is a real-valued function on $X$. Another important case is the following: when $Y=C(T, \mathbb{R})$ and 
$K=\{\phi \in C(T, \mathbb{R}): \phi(t) \geq 0 \forall t \in T\}$, (CIE) reduces to constraint systems of semi-infinite optimization problems; of course, one can also view the constraint systems in conic optimization problems as examples of (CIE). Hence conic inequality systems exist quite extensively. For such a kind of systems, we consider their error bounds. Let $S(\Phi, b, K)$ denote the solution set of (CIE); that is,

$$
S(\Phi, b, K):=\left\{x \in X: \Phi(x) \leq_{K} b\right\} .
$$

We say that (CIE) has a local error bound at $a \in S(\Phi, b, K)$ if there exist $\tau, \delta \in(0,+\infty)$ such that

$$
d(x, S(\Phi, b, K)) \leq \tau d(b, \Phi(x)+K) \quad \forall x \in B(a, \delta) .
$$

It is clear that (5) reduces to (1) when $Y=\mathbb{R}$ and $K=$ $\mathbb{R}_{+}$. The main aim of this paper is to extend the abovementioned Ioffe's classic result on error bounds to conic inequality (CIE) with $X, Y$ being any pair of Hilbert spaces in terms of proximal subdifferentials of vector-valued functions defined by proximal normal cone with a kind of variational behavior of "order two." In particular, we have improved Zheng and Ng's result in the Hilbert space setting.

\section{Preliminaries}

In this section, we summarize some fundamental tools of variational analysis and nonsmooth optimization using basically standard terminology and notation; see, for example, [13-15], for more details.

Let $X$ be a Banach space with topological dual $X^{*}$ and let $\overline{\mathbb{R}}:=\mathbb{R} \cup\{+\infty\}$. For a proper lower semicontinuous function $f: X \rightarrow \overline{\mathbb{R}}$, we denote by $\operatorname{dom}(f)$ and epi $(f)$ the domain and the epigraph of $f$, respectively; that is,

$$
\begin{gathered}
\operatorname{dom}(f):=\{x \in X: f(x)<+\infty\}, \\
\text { epi }(f):=\{(x, \alpha) \in X \times R: f(x) \leq \alpha\} .
\end{gathered}
$$

Throughout the paper, the symbol $\rightarrow$ always denotes the convergence relative to the distance $d(\cdot, \cdot)$ induced by the norm while the arrow $\stackrel{w^{*}}{\longrightarrow}$ signifies the weak ${ }^{*}$ convergence in the dual space $X^{*}$.

Recall that the Fréchet subdifferential of $f$ at $x$ is defined as (see $[15,16])$

$$
\begin{aligned}
\widehat{\partial} f(x):= & \left\{x^{*} \in X^{*}\right. \\
& \left.: \liminf _{u \rightarrow x} \frac{f(u)-f(x)-\left\langle x^{*}, u-x\right\rangle}{\|u-x\|} \geq 0\right\} .
\end{aligned}
$$

Let $\partial_{P} f(x)$ denote the proximal subdifferential of $f$ at $x$; that is,

$$
\begin{gathered}
\partial_{P} f(x):=\left\{x^{*} \in X^{*}: \exists \sigma, \eta \in(0,+\infty)\right. \\
\text { s.t. }\left\langle x^{*}, y-x\right\rangle \leq f(y)-f(x)+\sigma\|y-x\|^{2} \\
\forall y \in B(x, \eta)\} .
\end{gathered}
$$

For a closed subset $A$ of $X$ and $a \in A$, define, respectively, the Fréchet normal cone and the proximal normal cone $\widehat{N}(A, a)$ and $N_{P}(A, a)$ of $A$ to $a$ as

$$
\widehat{N}(A, a)=\widehat{\partial} \delta_{A}(a), \quad N_{P}(A, a)=\partial_{P} \delta_{A}(a),
$$

where $\delta_{A}$ denotes the indicator function of $A$ (i.e., $\delta_{A}(x)=0$ if $x \in A$ and $\delta_{A}(x)=+\infty$ otherwise). Clearly, $x^{*} \in N_{P}(A, a)$ if and only if each of the following two conditions holds.

(1) There exist $\sigma, r \in(0,+\infty)$ such that

$$
\left\langle x^{*}, x-a\right\rangle \leq \sigma\|x-a\|^{2} \quad \forall x \in B(a, r) \cap A .
$$

(2) There exists $\sigma^{\prime} \in(0,+\infty)$ such that

$$
\left\langle x^{*}, x-a\right\rangle \leq \sigma^{\prime}\|x-a\|^{2} \quad \forall x \in A .
$$

Let $N_{M}(A, a)$ denote the Mordukhovich (limiting) normal cone of $A$ to $a$; that is, $x^{*} \in N_{M}(A, a)$ if and only if there exist sequences $\left\{a_{n}\right\} \subset A$ and $\left\{x_{n}^{*}\right\} \subset X^{*}$ such that

$$
a_{n} \longrightarrow a, \quad x_{n}^{*} \stackrel{w^{*}}{\longrightarrow} x^{*}, \quad x_{n}^{*} \in N_{P}\left(A, a_{n}\right) .
$$

It is well known that

$$
\begin{gathered}
\widehat{\partial} f(x):=\left\{x^{*} \in X^{*}:\left(x^{*},-1\right) \in \widehat{N}(\operatorname{epi}(f),(x, f(x)))\right\}, \\
\partial_{P} f(x):=\left\{x^{*} \in X^{*}:\left(x^{*},-1\right) \in N_{P}(\operatorname{epi}(f),(x, f(x)))\right\} .
\end{gathered}
$$

Let $Y$ be a Banach space and let $K \subset Y$ be a closed convex cone, which defines a preorder $\leq_{K}$ in $Y$ as follows: $y_{1} \leq_{K} y_{2} \Leftrightarrow y_{2}-y_{1} \in K$. Let

$$
\begin{gathered}
K^{+}:=\left\{y^{*} \in Y^{*}:\left\langle y^{*}, y\right\rangle \geq 0 \forall y \in K\right\}, \\
\mathscr{I}_{K^{+}}:=\left\{y^{*} \in K^{+}:\left\|y^{*}\right\|=1\right\} ;
\end{gathered}
$$

usually $K^{+}$is called the dual cone of $K$. Let $\infty_{Y}$ denote an abstract infinity and $Y^{\bullet}=Y \cup\left\{\infty_{Y}\right\}$. For a vector-valued function $f: X \rightarrow Y^{\bullet}$, the epigraph of $f$ with respect to the ordering cone $K$ is defined by

$$
\operatorname{epi}_{K}(f):=\left\{(x, y) \in X \times Y: f(x) \leq_{K} y\right\} .
$$

In the vector-valued setting, in view of (13), we will use the following kinds of subdifferentials of $f$ :

$$
\begin{aligned}
& \widehat{\partial}_{K} f(x)=\left\{x^{*} \in X^{*}\right. \\
&\left.:\left(x^{*},-\mathscr{I}_{K^{+}}\right) \cap \widehat{N}\left(\operatorname{epi}_{K}(f),(x, f(x))\right) \neq \emptyset\right\}, \\
& \partial_{K}^{P} f(x)=\left\{x^{*} \in X^{*}\right.\left.:\left(x^{*},-\mathscr{I}_{K^{+}}\right) \cap N_{P}\left(\operatorname{epi}_{K}(f),(x, f(x))\right) \neq \emptyset\right\}, \\
& \partial_{K}^{M} f(x)=\left\{x^{*} \in X^{*}\right. \\
&\left.:\left(x^{*},-\mathscr{I}_{K^{+}}\right) \cap N_{M}\left(\operatorname{epi}_{K}(f),(x, f(x))\right) \neq \emptyset\right\} .
\end{aligned}
$$


In the special case when $Y=\mathbb{R}$ and $K=\mathbb{R}^{+}$, we have $\mathscr{I}_{K^{+}}=$ $\{1\}$,

$$
\widehat{\partial}_{K} f(x)=\widehat{\partial} f(x), \quad \partial_{K}^{P} f(x)=\partial_{P} f(x) .
$$

The following lemma will be useful in our analysis later.

Lemma 1. Let $X, Y$ be Hilbert spaces with $K \subset Y$ being a closed convex cone and suppose that $f: X \rightarrow Y^{\bullet}$ is a function such that $\operatorname{epi}_{K}(f)$ is closed. Then, for any $x \in \operatorname{dom}(f)$ and $y \in K$,

$$
N_{P}\left(\operatorname{epi}_{K}(f),(x, f(x)+y)\right) \subset N_{P}\left(\operatorname{epi}_{K}(f),(x, f(x))\right) .
$$

Proof. Let $\left(x^{*}, y^{*}\right) \in N_{P}\left(\operatorname{epi}_{K}(f),(x, f(x)+y)\right)$. Then it follows from (11) that there exists $\sigma \in(0,+\infty)$ such that

$$
\begin{aligned}
& \left\langle\left(x^{*}, y^{*}\right),\left(u^{\prime}, v^{\prime}\right)-(x, f(x)+y)\right\rangle \\
& \quad \leq \sigma\left\|\left(u^{\prime}, v^{\prime}\right)-(x, f(x)+y)\right\|^{2} \quad \forall\left(u^{\prime}, v^{\prime}\right) \in \operatorname{epi}_{K}(f) .
\end{aligned}
$$

Noting that for any $y \in K$

$$
(u, v) \in \operatorname{epi}_{K}(f) \Longrightarrow(u, v+y) \in \operatorname{epi}_{K}(f)
$$

and that

$$
(u, v)-(x, f(x))=(u, v+y)-(x, f(x)+y),
$$

one has

$$
\begin{aligned}
& \left\langle\left(x^{*}, y^{*}\right),(u, v)-(x, f(x))\right\rangle \\
& \quad \leq \sigma\|(u, v)-(x, f(x))\|^{2} \quad \forall(u, v) \in \mathrm{epi}_{K}(f) .
\end{aligned}
$$

This means that $\left(x^{*}, y^{*}\right) \in N_{P}\left(\operatorname{epi}_{K}(f),(x, f(x))\right)$, which implies in turn that

$$
N_{P}\left(\operatorname{epi}_{K}(f),(x, f(x)+y)\right) \subset N_{P}\left(\operatorname{epi}_{K}(f),(x, f(x))\right),
$$

and thus completes the proof of the lemma.

In establishing our main results, it will be crucial to present the following lemmas (cf. Chapter 1, Proposition 2.11 and Theorem 6.1 in [14] and Chapter 8, Theorem 8.3.3 in [16]).

Lemma 2. Let $X$ be a Hilbert space and let $f, g: X \rightarrow \mathbb{R} \cup$ $\{+\infty\}$ be proper lower semicontinuous and suppose that $g$ is twice continuously differentiable at $x_{0} \in \operatorname{dom} f$. Then

$$
\partial_{P}(f+g)\left(x_{0}\right)=\partial_{P} f\left(x_{0}\right)+g^{\prime}\left(x_{0}\right)
$$

where $g^{\prime}\left(x_{0}\right)$ denotes the derivative of $g$ at $x_{0}$.

Lemma 3. Let $A$ be a nonempty closed subset of a Hilbert space $X$ and let $x \in X \backslash A$ be such that $\partial_{P} d(\cdot, A)(x) \neq \emptyset$. Then there exists $a \in A$ satisfying the following properties.

(i) The set of closest points $P_{A}(x)$ in $A$ to $x$ is the singleton $\{a\}$. (ii) $d(\cdot, A)$ is Fréchet differentiable at $x$ and

$$
\partial_{P} d(\cdot, A)(x)=\left\{d^{\prime}(\cdot, A)(x)\right\}=\left\{\frac{x-a}{\|x-a\|}\right\} .
$$

(iii) $x-a \in N_{P}(A, a)$.

Lemma 4. Let $X$ be a Hilbert space and let $f: X \rightarrow \mathbb{R} \cup\{+\infty\}$ be a proper lower semicontinuous function. Let $x_{0} \in X$ and $\varepsilon \in(0,+\infty)$ be such that $f\left(x_{0}\right)<\inf _{x \in X} f(x)+\varepsilon$. Then, for any $>0$, there exist $y$ and $z$ in $X$ with

$$
\begin{gathered}
\left\|z-x_{0}\right\|<\lambda, \quad\|y-z\|<\lambda \\
f(y) \leq f\left(x_{0}\right),
\end{gathered}
$$

having the following property:

$$
f(z)+\frac{\varepsilon}{\lambda^{2}}\|y-z\|^{2} \leq f(x)+\frac{\varepsilon}{\lambda^{2}}\|x-y\|^{2} \quad \forall x \in X .
$$

\section{Main Results}

As an extension of Ioffe's classic results on error bounds to conic inequality in Asplund spaces, Zheng and $\mathrm{Ng}$ [12] proved Theorem I.

Theorem I. Let X, $Y$ be Asplund spaces with $K \subset Y$ being a closed convex cone. Let $\Phi: X \rightarrow Y^{\bullet}$ be a function such that its $K$-epigraph is closed. Let $a \in S(\Phi, b, K)$ and $\eta, r \in(0,+\infty)$ be such that

$$
\eta \leq d\left(0, \widehat{\partial}_{K} \Phi(x)\right) \quad \forall x \in B(a, r) \backslash S(\Phi, b, K) .
$$

Then conic inequality (CIE) has a local error bound at a.

It is well known that $\partial_{K}^{P} \Phi(x) \subset \widehat{\partial}_{K} \Phi(x)$ for any $x \in$ dom $\Phi$. So condition (28) will be much easier to be satisfied if $\widehat{\partial}_{K} \Phi(x)$ is replaced by $\partial_{K}^{P} \Phi(x)$. Taking this fact into account, Theorem 6 provides a sharper result with $\widehat{\partial}_{K} \Phi$ replaced by $\partial_{K}^{P} \Phi$ and gives a relationship between the modulus of error bound and corresponding radius which were not mentioned in Theorem I. Its proof, which is slightly different from the one of Theorem I in [12], is based on a smooth variational principle rather than the Ekeland variational principle. Since the proof of Theorem 6 proceeds by contradiction, we give the following proposition to describe quantitative properties for a point that violates (5).

Proposition 5. Let $X, Y$ be Hilbert spaces with $K \subset Y$ being a closed convex cone. Let $\Phi: X \rightarrow Y^{\bullet}$ be a function such that its K-epigraph is closed. Let $a \in S(\Phi, b, K), x_{0} \in X$, and $\tau \in(0,+\infty)$ be such that

$$
\tau d\left(b, \Phi\left(x_{0}\right)+K\right)<d\left(x_{0}, S(\Phi, b, K)\right) .
$$


Then there exist $(u, v) \in \operatorname{epi}_{K}(\Phi),(\widetilde{u}, \widetilde{v}) \in X \times Y$ satisfying the following properties:

$$
\begin{gathered}
\|u-\widetilde{u}\|<d\left(x_{0}, S(\Phi, b, K)\right), \\
\|v-\widetilde{v}\|<d\left(x_{0}, S(\Phi, b, K)\right), \\
\left\|u-x_{0}\right\|<\left\|x_{0}-a\right\|, \quad u \notin S(\Phi, b, K), \\
(0,0) \in\{0\} \times\left\{\frac{v-b}{\|v-b\|}\right\}+\frac{2(u-\widetilde{u}, v-\widetilde{v})}{\tau d\left(x_{0}, S(\Phi, b, K)\right)} \\
+N_{P}\left(\operatorname{epi}_{K}(\Phi),(u, v)\right) .
\end{gathered}
$$

Proof. It follows from (29) that there exists $y_{0} \in K$ such that

$$
\tau\left\|b-\Phi\left(x_{0}\right)-y_{0}\right\|<d\left(x_{0}, S(\Phi, b, K)\right) .
$$

Define the function $\phi$ by $\phi(x, y):=\|y-b\|+\delta_{\text {epi }_{K}(\Phi)}(x, y)$ for all $(x, y) \in X \times Y$. Then $\phi$ is lower semicontinuous (due to the closedness of $\left.\operatorname{epi}_{K}(\Phi)\right)$ and

$$
\begin{aligned}
\phi\left(x_{0}, \Phi\left(x_{0}\right)+y_{0}\right)< & \frac{d\left(x_{0}, S(\Phi, b, K)\right)}{\tau} \\
\leq & \inf \{\phi(x, y):(x, y) \in X \times Y\} \\
& +\frac{d\left(x_{0}, S(\Phi, b, K)\right)}{\tau} .
\end{aligned}
$$

This and Lemma 4 imply that there exist $(\tilde{u}, \widetilde{v}),(u, v) \in X \times Y$ such that

$$
\begin{gathered}
\left(\left\|u-x_{0}\right\|^{2}+\left\|v-\Phi\left(x_{0}\right)-y_{0}\right\|^{2}\right)^{1 / 2}<d\left(x_{0}, S(\Phi, b, K)\right) \\
\left(\|u-\widetilde{u}\|^{2}+\|v-\widetilde{v}\|^{2}\right)^{1 / 2}<d\left(x_{0}, S(\Phi, b, K)\right) \\
\phi(u, v)+\frac{\|(u, v)-(\widetilde{u}, \widetilde{v})\|^{2}}{\tau d\left(x_{0}, S(\Phi, b, K)\right)} \\
\leq \phi(x, y)+\frac{\|(x, y)-(\widetilde{u}, \widetilde{v})\|^{2}}{\tau d\left(x_{0}, S(\Phi, b, K)\right)} \\
\forall(x, y) \in X \times Y
\end{gathered}
$$

From (36), it is easy to verify that (30) holds. Applying (35) and $a \in S(\Phi, b, K)$ leads us to the following:

$$
\left\|u-x_{0}\right\|<d\left(x_{0}, S(\Phi, b, K)\right) \leq\left\|x_{0}-a\right\|,
$$

which also implies that

$$
u \notin S(\Phi, b, K),
$$

and thus verifies (31), while (37) together with $(a, b) \in$ $\operatorname{epi}_{K}(\Phi)$ gives us $(u, v) \in \operatorname{dom}(\phi)=\operatorname{epi}_{K}(\Phi)$ and so $\Phi(u) \leq_{K} v$. It follows from this and (39) that

$$
v \neq b
$$

Furthermore, (37) implies that

$$
(0,0) \in \partial_{P}\left(\psi+\delta_{\operatorname{epi}_{K}(\Phi)}\right)(u, v)
$$

where

$$
\psi(x, y):=\|y-b\|+\frac{\|(x, y)-(\widetilde{u}, \widetilde{v})\|^{2}}{\tau d\left(x_{0}, S(\Phi, b, K)\right)} \quad \forall(x, y) \in X \times Y .
$$

It follows from (40) and Lemma 3 that $\psi$ is twice continuously differentiable at $(u, v)$ and

$$
\psi^{\prime}(u, v)=\{0\} \times\left\{\frac{v-b}{\|v-b\|}\right\}+\frac{2(u-\widetilde{u}, v-\widetilde{v})}{\tau d\left(x_{0}, S(\Phi, b, K)\right)} .
$$

So applying Lemma 2 to (41) leads us to the inclusions

$$
\begin{aligned}
(0,0) \in & \{0\} \times\left\{\frac{v-b}{\|v-b\|}\right\}+\frac{2(u-\widetilde{u}, v-\widetilde{v})}{\tau d\left(x_{0}, S(\Phi, b, K)\right)} \\
& +\partial_{P} \delta_{\operatorname{epi}_{K}(\Phi)}(u, v) \\
= & \{0\} \times\left\{\frac{v-b}{\|v-b\|}\right\}+\frac{2(u-\widetilde{u}, v-\widetilde{v})}{\tau d\left(x_{0}, S(\Phi, b, K)\right)} \\
& +N_{P}\left(\operatorname{epi}_{K}(\Phi),(u, v)\right),
\end{aligned}
$$

which justifies (32) and thus completes the proof of the proposition.

With the preparation that we have done, the proof of our main result is now straightforward.

Theorem 6. Let $X, Y$ be Hilbert spaces with $K \subset Y$ being $a$ closed convex cone. Let $\Phi: X \rightarrow Y^{\bullet}$ be a function such that its $K$-epigraph is closed. Let $a \in S(\Phi, b, K)$ and $\eta, r \in(0,+\infty)$ be such that

$$
\eta \leq d\left(0, \partial_{K}^{P} \Phi(x)\right) \quad \forall x \in B(a, r) \backslash S(\Phi, b, K) .
$$

Then, one has

$$
\begin{aligned}
& d(x, S(\Phi, b, K)) \\
& \quad \leq\left(2+\frac{2}{\eta}\right) d(b, \Phi(x)+K) \quad \forall x \in B\left(a, \frac{r}{2}\right) .
\end{aligned}
$$

Proof. The proof proceeds by contradiction. Namely, suppose to the contrary that (46) is not true; then, there exists $x_{0} \in$ $B(a, r / 2)$ such that

$$
d\left(x_{0}, S(\Phi, b, K)\right)>\left(2+\frac{2}{\eta}\right) d\left(b, \Phi\left(x_{0}\right)+K\right)
$$

and consequently it follows from Proposition 5 that there exist $(u, v) \in \operatorname{epi}_{K}(\Phi),(\tilde{u}, \widetilde{v}) \in X \times Y$ such that (30), (31), and (32) hold. Then, one has

$$
\begin{aligned}
\|u-a\| & \leq\left\|u-x_{0}\right\|+\left\|x_{0}-a\right\| \\
& <\left\|x_{0}-a\right\|+\left\|x_{0}-a\right\|=2\left\|x_{0}-a\right\|<r,
\end{aligned}
$$


which implies

$$
u \in B(a, r) \backslash S(\Phi, b, K) .
$$

Since $(u, v) \in \operatorname{epi}_{K}(\Phi)$, we can take $z \in K$ such that $v=$ $\Phi(u)+z$. Then, by Lemma 1 , one has

$$
N_{P}\left(\operatorname{epi}_{K}(\Phi),(u, \Phi(u)+z)\right) \subset N_{P}\left(\operatorname{epi}_{K}(\Phi),(u, \Phi(u))\right) .
$$

It follows from (32) and (50) that

$$
\begin{aligned}
& \left(-\frac{u-\widetilde{u}}{(1+1 / \eta) d\left(x_{0}, S(\Phi, b, \mathrm{~K})\right)},\right. \\
& \left.\quad-\frac{v-b}{\|v-b\|}-\frac{v-\widetilde{v}}{(1+1 / \eta) d\left(x_{0}, S(\Phi, b, K)\right)}\right) \\
& \quad \in N_{P}\left(\operatorname{epi}_{K}(\Phi),(u, \Phi(u))\right) .
\end{aligned}
$$

Noting that $(u, \Phi(u))+\{0\} \times K \subset \operatorname{epi}_{K}(\Phi)$, this implies that $((v-b) /\|v-b\|)+\left((v-\widetilde{v}) /(1+1 / \eta) d\left(x_{0}, S(\Phi, b, K)\right)\right) \in K^{+}$. Indeed, due to the definition of proximal normal cone, there exists $\sigma \in(0,+\infty)$ such that

$$
\begin{aligned}
& \left\langle\left(-\frac{u-\tilde{u}}{(1+1 / \eta) d\left(x_{0}, S(\Phi, b, K)\right)},\right.\right. \\
& \left.\quad-\frac{v-b}{\|v-b\|}-\frac{v-\widetilde{v}}{(1+1 / \eta) d\left(x_{0}, S(\Phi, b, K)\right)}\right), \\
& \left.\quad\left(u^{\prime}, v^{\prime}\right)-(u, \Phi(u))\right\rangle \\
& \leq \sigma\left\|\left(u^{\prime}, v^{\prime}\right)-(u, \Phi(u))\right\|^{2}
\end{aligned}
$$

for all $\left(u^{\prime}, v^{\prime}\right) \in \operatorname{epi}_{K}(\Phi)$.

Namely,

$$
\begin{aligned}
& \left(\left\langle-\frac{u-\tilde{u}}{(1+1 / \eta) d\left(x_{0}, S(\Phi, b, K)\right)}, u^{\prime}-u\right\rangle\right. \\
& -\left\langle\frac{v-b}{\|v-b\|}+\frac{v-\widetilde{v}}{(1+1 / \eta) d\left(x_{0}, S(\Phi, b, K)\right)}\right. \\
& \left.\left.v^{\prime}-\Phi(u)\right\rangle\right) \\
& \times\left(\left\|\left(u^{\prime}, v^{\prime}\right)-(u, \Phi(u))\right\|^{2}\right)^{-1} \leq \sigma
\end{aligned}
$$

for all $\left(u^{\prime}, v^{\prime}\right) \in \operatorname{epi}_{K}(\Phi)$.

Setting $u^{\prime}=u$ in the above inequality, one has

$$
\begin{aligned}
& -\left\langle\frac{v-b}{\|v-b\|}+\frac{v-\widetilde{v}}{(1+1 / \eta) d\left(x_{0}, S(\Phi, b, K)\right)},\right. \\
& \left.\quad v^{\prime}-\Phi(u)\right\rangle \\
& \times\left(\left\|v^{\prime}-\Phi(u)\right\|^{2}\right)^{-1} \leq \sigma .
\end{aligned}
$$

Noting that $v^{\prime}-\Phi(u) \in K$ and $K$ is a cone, one has

$$
\left\langle\frac{v-b}{\|v-b\|}+\frac{v-\widetilde{v}}{(1+1 / \eta) d\left(x_{0}, S(\Phi, b, K)\right)}, v^{\prime}-\Phi(u)\right\rangle \geq 0,
$$

which means that

$$
\frac{v-b}{\|v-b\|}+\frac{v-\widetilde{v}}{(1+1 / \eta) d\left(x_{0}, S(\Phi, b, K)\right)} \in K^{+}
$$

Let

$$
\begin{aligned}
x^{*}:= & \left(-\frac{u-\tilde{u}}{(1+1 / \eta) d\left(x_{0}, S(\Phi, b, K)\right)}\right) \\
& \times \| \frac{v-b}{\|v-b\|}
\end{aligned}
$$

$$
+\frac{(v-\tilde{v})}{(1+1 / \eta) d\left(x_{0}, S(\Phi, b, K)\right)} \|^{-1},
$$$$
z^{*}:=\left(\frac{v-b}{\|v-b\|}\right.
$$

$$
\begin{aligned}
& \left.+\frac{v-\widetilde{v}}{(1+1 / \eta) d\left(x_{0}, S(\Phi, b, K)\right)}\right) \\
& \times \| \frac{v-b}{\|v-b\|}
\end{aligned}
$$

$$
+\frac{v-\widetilde{v}}{(1+1 / \eta) d\left(x_{0}, S(\Phi, b, K)\right)} \|^{-1} .
$$

Then $\left(x^{*}-z^{*}\right) \in N_{P}\left(\operatorname{epi}_{K}(\Phi),(u, \Phi(u))\right)$ and so $x^{*} \in \partial_{K}^{P} \Phi(u)$. Combining this with (30) implies that

$$
\begin{aligned}
& d\left(0, \partial_{K}^{P} \Phi(u)\right) \\
& \quad \leqslant\left\|x^{*}\right\| \leq \frac{\|u-\widetilde{u}\| /(1+1 / \eta) d\left(x_{0}, S(\Phi, b, K)\right)}{1-\left(\|v-\widetilde{v}\| /(1+1 / \eta) d\left(x_{0}, S(\Phi, b, K)\right)\right)} \\
& \quad<\frac{1 /(1+1 / \eta)}{1-(1 /(1+1 / \eta))} \\
& \quad=\eta,
\end{aligned}
$$

which contradicts (45) and (49). The proof is complete.

Letting $r \rightarrow+\infty$ in Theorem 6 , we have the following global error bound result.

Corollary 7. Let $X, Y$ be Hilbert spaces with $K \subset Y$ being a closed convex cone. Let $\Phi: X \rightarrow Y^{\bullet}$ be a function such that its $K$-epigraph is closed. Let $a \in S(\Phi, b, K)$ and $\eta, r \in(0,+\infty)$ be such that

$$
\eta \leq d\left(0, \partial_{K}^{P} \Phi(x)\right) \quad \forall x \in X \backslash S(\Phi, b, K) .
$$


Then, one has

$$
d(x, S(\Phi, b, K)) \leq\left(2+\frac{2}{\eta}\right) d(b, \Phi(x)+K) \quad \forall x \in X .
$$

Taking Theorems I and 6 and Corollary 7 into consideration, we arrive at the following remarks.

Remark 8. (1) Since the class of Asplund space is more extensive than that of Hilbert space from the framework of spaces, Theorem I has a wider range of applications than Theorem 6.

(2) The subdifferential $\partial_{K}^{P} \Phi(x)$ in Theorem 6 expresses a kind of variational behavior of "order two," while $\widehat{\partial}_{K} \Phi(x)$ in Theorem I is of "order one." In general, $\partial_{K}^{P} \Phi(x)$ is smaller than $\widehat{\partial}_{K} \Phi(x)$, and, furthermore, $\operatorname{dom}\left(\partial_{K}^{P} \Phi\right)$ may have measure zero even for a smooth function. In [17], Clarke et al. have constructed a $C^{1}$ function on $\mathbb{R}$ whose proximal subgradient is nonempty on a set that is small in the sense of measure and category. For the sake of convenience, we present this interesting result to show this kind of somewhat surprising phenomenon as follows.

Let $g$ be the (continuous) function of period 4 which satisfies $g(x)=x+1$ for $-2 \leq x \leq 0, g(x)=-x+1$ for $0 \leq x \leq 2$, and define

$$
\varphi(x):=\sum_{n=1}^{\infty} 2^{-n} g\left(2^{2^{n}} x\right) .
$$

Clearly $\varphi$ is continuous, and thus $f(\cdot)$ defined by $f(x):=$ $\int_{0}^{x} \varphi(t) d t$ is $C^{1}$ on $\mathbb{R}$. Following the excellent proof of Clarke, we arrive at the conclusion that both $\operatorname{dom}\left(\partial_{P} f\right)$ and $\operatorname{dom}\left(\partial_{P}(-f)\right)$ have measure zero. For more details, we refer the reader to Theorem 6.1 in [17].

(3) To our knowledge, we do not know whether the assumption of Hilbert spaces in Theorem 6 and Corollary 7 can be extended to more general Banach spaces or not, for example, whether these results can be extended to $\ell^{p}(1<$ $p<+\infty)$ spaces.

Under the assumption somewhat stronger than that for Theorem 6, we have the following stability version regarding local error bounds for (CIE).

Theorem 9. Let $X, Y$ be Hilbert spaces and suppose that $\Phi$ : $X \rightarrow Y^{\bullet}$ is continuous and that $K \subset Y$ is a closed convex cone such that $\operatorname{int}(K) \neq \emptyset$, where $\operatorname{int}(\cdot)$ denotes the interior. Let $a \in X$ be such that $0 \notin \partial_{K}^{M} \Phi(a)$. Then, there exists $\gamma>0$ such that, for any $b \in \Phi(B(a, \gamma))+K$, the corresponding conic inequality (CIE) has a local error bound at each $x \in B(a, \gamma) \cap S(\Phi, b, K)$.

Proof. By Theorem 6, it suffices to show that there exist $r, \eta>$ 0 such that $d\left(0, \partial_{K}^{P} \Phi(x)\right) \geq \eta$ for all $x \in B(a, r)$. If it is not the case, we can find a sequence $\left\{x_{n}\right\}$ in $X$ converging to $a$ and satisfying

$$
d\left(0, \partial_{K}^{P} \Phi\left(x_{n}\right)\right)<\frac{1}{n} \quad \forall n \in \mathbb{N}
$$

By the definition of $\partial_{K}^{P} \Phi$, this means that there exist $y_{n}^{*} \in K^{+}$ and $x_{n}^{*} \in X^{*}$ such that

$$
\begin{gathered}
\left\|y_{n}^{*}\right\|=1, \quad\left\|x_{n}^{*}\right\|<\frac{1}{n}, \\
\left(x_{n}^{*},-y_{n}^{*}\right) \in N_{P}\left(\operatorname{epi}_{K}(\Phi),\left(x_{n}, \Phi\left(x_{n}\right)\right)\right) .
\end{gathered}
$$

Without loss of generality, we assume that $\left\{y_{n}^{*}\right\}$ converges to $y^{*} \in K^{+}$with the weak $^{*}$ topology (taking a subsequence if necessary). Taking the continuity assumption of $\Phi$ into consideration, it follows from (63) that

$$
\left(0,-y^{*}\right) \in N_{M}\left(\operatorname{epi}_{K}(\Phi),(a, \Phi(a))\right) .
$$

We claim that $y^{*} \neq 0$. Granting this, one has $\left(0,-y^{*} /\left\|y^{*}\right\|\right) \in$ $N_{M}\left(\operatorname{epi}_{K}(\Phi),(a, \Phi(a))\right)$, which contradicts the assumption that $0 \notin \partial_{K}^{M} \Phi(a)$. It remains to show that $y^{*} \neq 0$. Since $\operatorname{int}(K) \neq \emptyset$, there exist $k \in K$ and $\delta>0$ such that $k+\delta B_{Y} \subset K$. Hence,

$$
\begin{aligned}
0 & \leq \inf \left\{\left\langle y_{n}^{*}, y\right\rangle: y \in k+\delta B_{Y}\right\} \\
& =\left\langle y_{n}^{*}, k\right\rangle-\delta\left\|y_{n}^{*}\right\|=\left\langle y_{n}^{*}, k\right\rangle-\delta,
\end{aligned}
$$

and so $\delta \leq\left\langle y_{n}^{*}, k\right\rangle$. Since $y_{n}^{*} \stackrel{w^{*}}{\longrightarrow} y^{*}, \delta \leq\left\langle y^{*}, k\right\rangle$. This shows that $y^{*} \neq 0$, which completes the proof.

It is important to note that $\operatorname{int}(K) \neq \emptyset$ plays an important role in the validity of Theorem 9 , as the following example shows. Hence the assumption of $\operatorname{int}(K) \neq \emptyset$ in Theorem 9 is not superfluous.

Example 10 (failure of having a local error bound). Let $X=Y=\ell^{2}, K=\ell_{+}^{2}, a=b=0$, and $\Phi(x)=$ $\left(x_{1} / 2, x_{2} / 2^{2}, \ldots, x_{n} / 2^{n}, \ldots\right)$ for all $x \in \ell^{2}$. Then $\Phi$ is continuous, $0 \notin \partial_{K}^{M} \Phi(a)$ and $S(\Phi, b, K)=\ell_{-}^{2}$.

It is well known and easy to check that $\operatorname{int}(K)=\operatorname{int}\left(\ell_{+}^{2}\right)=$ $\emptyset$. Take a sequence $\left\{e_{n}\right\}$ in $\ell^{2}$, where

$$
e_{n}=(\underbrace{0, \ldots, 0, \frac{1}{n}}_{n}, 0, \ldots) \text {. }
$$

Then $\left\{e_{n}\right\}$ converges to $a=0 \in S(\Phi, b, K)$ and $\Phi\left(e_{n}\right)=$ $(\underbrace{0, \ldots, 0,1 / 2^{n} \cdot n}_{n}, 0, \ldots)$.

$$
\begin{aligned}
d\left(b, \Phi\left(e_{n}\right)+K\right) & =d\left(-\Phi\left(e_{n}\right), K\right) \\
& =d\left(-\Phi\left(e_{n}\right), \ell_{+}^{2}\right) \\
& =\inf _{y \in \ell_{+}^{2}}\left\|-\Phi\left(e_{n}\right)-y\right\| \\
& =\inf _{y \in \ell_{+}^{2}}\left\|(\underbrace{0, \ldots, 0,-\frac{1}{2^{n} \cdot n}}_{n}, 0, \ldots)-y\right\| \\
& =\frac{1}{2^{n} \cdot n}
\end{aligned}
$$


and that

$$
\begin{aligned}
d\left(e_{n}, S(\Phi, b, K)\right) & =d\left(e_{n}, \ell_{-}^{2}\right) \\
& =\inf _{x \in \ell_{-}^{2}}\left\|e_{n}-x\right\| \\
& =\inf _{x \in \ell_{-}^{2}}\left\|(\underbrace{0, \ldots, 0, \frac{1}{n}}_{n}, 0, \ldots)-x\right\| \\
& =\frac{1}{n},
\end{aligned}
$$

it follows that

$$
\begin{aligned}
& n d\left(b, \Phi\left(e_{n}\right)+K\right) \\
& \quad=\frac{1}{2^{n}}<\frac{1}{n}=d\left(e_{n}, S(\Phi, b, K)\right) \quad \forall n \in \mathbb{N} .
\end{aligned}
$$

Hence the conic inequality (CIE) does not have a local error bound at $a$. This situation occurs, of course, because $\operatorname{int}(K)$ is empty.

\section{Conflict of Interests}

The authors declare that there is no conflict of interests regarding the publication of this paper.

\section{Acknowledgments}

This research was supported by the National Natural Science Foundation of China (Grant nos. 11371312 and 11261067) and IRTSTYN.

\section{References}

[1] A. J. Hoffman, "On approximate solutions of systems of linear inequalities," Journal of Research of the National Bureau of Standards, vol. 49, pp. 263-265, 1952.

[2] D. Azé and J. N. Corvellec, "Characterizations of error bounds for lower semicontinuous functions on metric spaces," ESAIM. Control, Optimisation and Calculus of Variations, vol. 10, no. 3, pp. 409-425, 2004.

[3] A. D. Ioffe, "Metric regularity and subdifferential calculus," Russian Mathematical Surveys, vol. 55, no. 3, pp. 501-558, 2000.

[4] A. S. Lewis and J. S. Pang, "Error bounds for convex inequality systems," in Generalized Convexity, Generalized Monotonicity: Recent Results, J. P. Crouzeix, J. E. Martinez-Legaz, and M. Volle, Eds., vol. 27 of Nonconvex Optimization and Its Applications, pp. 75-110, Kluwer Academic Publishers, Dordrecht, The Netherlands, 1998.

[5] K. F. Ng and X. Y. Zheng, "Error bounds for lower semicontinuous functions in normed spaces," SIAM Journal on Optimization, vol. 12, no. 1, pp. 1-17, 2001.

[6] H. van Ngai and M. Théra, "Error bounds for systems of lower semicontinuous functions in Asplund spaces," Mathematical Programming, vol. 116, no. 1-2, pp. 397-427, 2009.

[7] J. S. Pang, "Error bounds in mathematical programming," Mathematical Programming B, vol. 79, no. 1-3, pp. 299-332, 1997.
[8] Z. Wu and J. J. Ye, "On error bounds for lower semicontinuous functions," Mathematical Programming, vol. 92, no. 2, pp. 301314, 2002.

[9] C. Zalinescu, "Weak sharp minima, well-behaving functions and global error bounds for convex inequalities in Banach spaces," in Proceedings of the 12th Baikal International Conference on Optimization Methods and Their Applications, pp. 272284, Irkutsk, Russia, 2001.

[10] A. D. Ioffe, "Regular points of Lipschitz functions," Transactions of the American Mathematical Society, vol. 251, pp. 61-69, 1979.

[11] X. Y. Zheng and K. F. Ng, "Metric subregularity and calmness for nonconvex generalized equations in Banach spaces," SIAM Journal on Optimization, vol. 20, no. 5, pp. 2119-2136, 2010.

[12] X. Y. Zheng and K. F. Ng, "Error bound for conic inequality," Vietnam Journal of Mathematics. In press.

[13] F. H. Clarke, Optimization and Nonsmooth Analysis, John Wiley \& Sons, New York, NY, USA, 1983.

[14] F. H. Clarke, Yu. S. Ledyaev, R. J. Stern, and P. R. Wolenski, Nonsmooth Analysis and Control Theory, Springer, New York, NY, USA, 1998.

[15] B. S. Mordukhovich, Variational Analysis and Generalized Differentiation. I/II, Springer, Berlin, Germany, 2006.

[16] W. Schirotzek, Nonsmooth Analysis, Springer, Berlin, Germany, 2007.

[17] F. H. Clarke, Yu. S. Ledyaev, and P. R. Wolenski, "Proximal analysis and minimization principles," Journal of Mathematical Analysis and Applications, vol. 196, no. 2, pp. 722-735, 1995. 


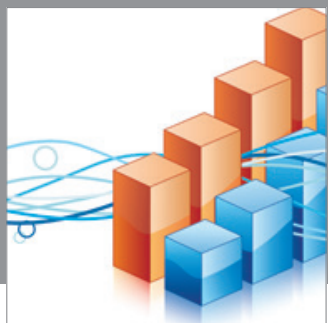

Advances in

Operations Research

mansans

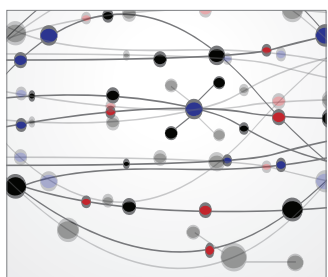

The Scientific World Journal
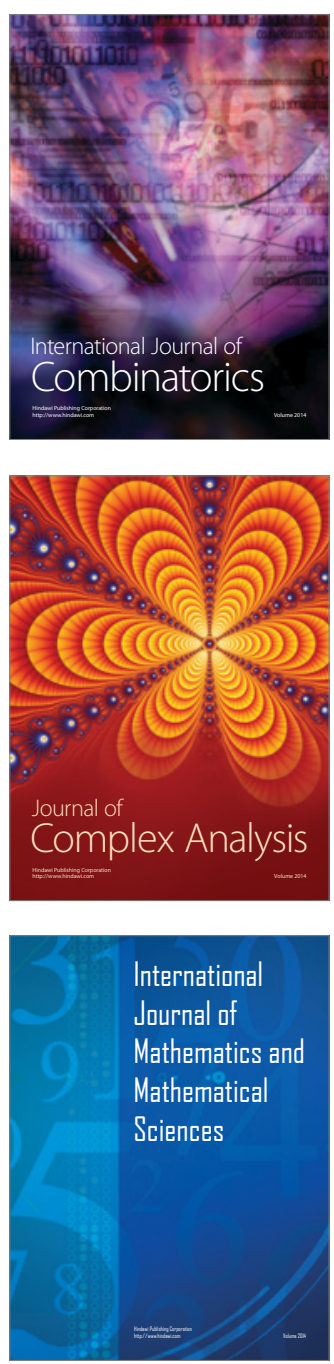
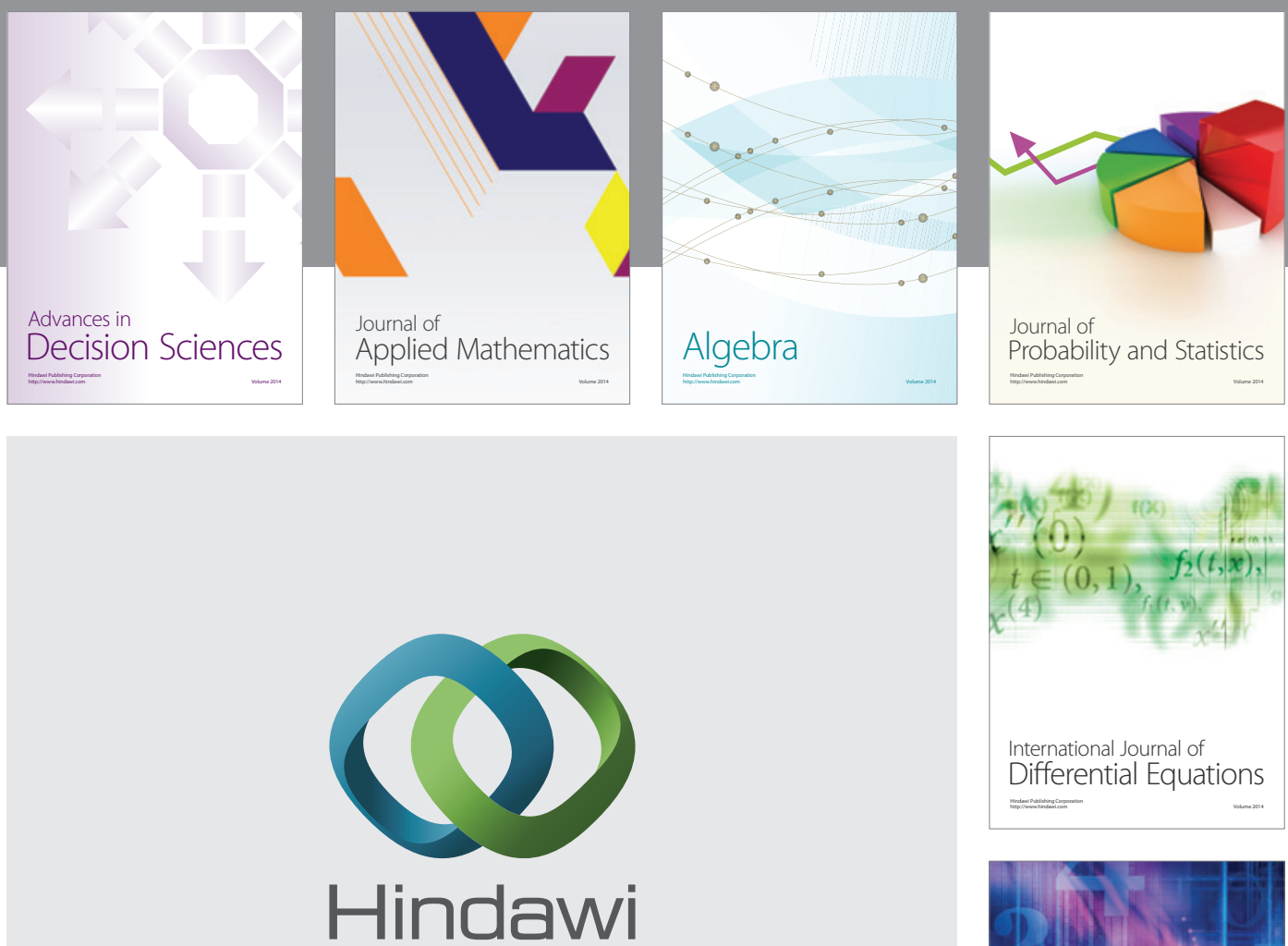

Submit your manuscripts at http://www.hindawi.com
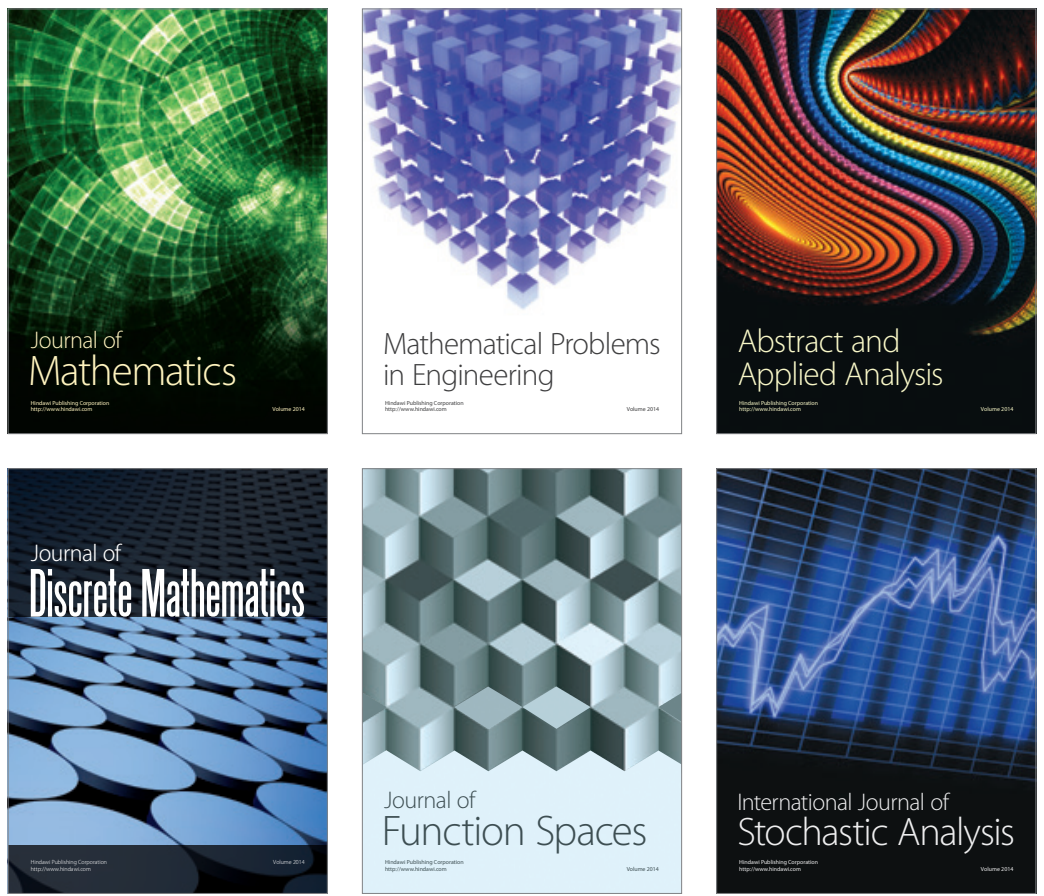

Journal of

Function Spaces

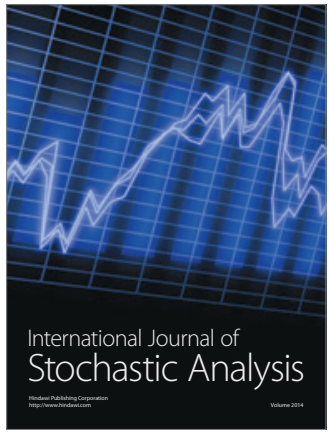

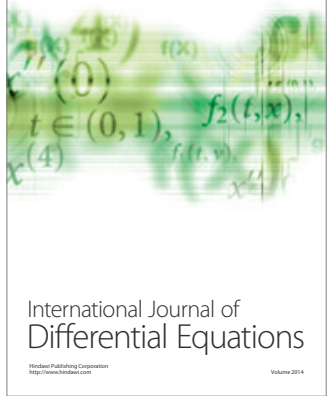
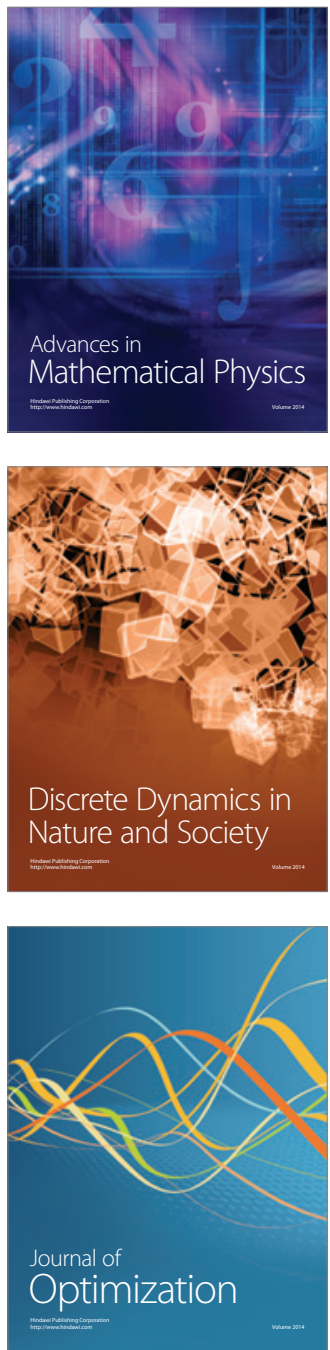